\title{
A new result on the existence of periodic solutions for Rayleigh equations with a singularity of repulsive type
}

\section{Lijuan Chen ${ }^{*}$ and Shiping Lu}

\section{"Correspondence:}

cljung@sohu.com

College of Math \& Statistics, Nanjing

University of Information Science \&

Technology, Nanjing, 210044, China

\begin{abstract}
In this paper, the problem of the existence of periodic solutions is studied for the second-order differential equations with a singularity of repulsive type,

$$
x^{\prime \prime}(t)+f\left(x^{\prime}(t)\right)+\varphi(t) x(t)-\frac{1}{x^{r}(t)}=h(t)
$$

where $\varphi$ and $h$ are $T$-periodic functions. By using topological degree theory, a new result on the existence of positive periodic solutions is obtained. The interesting thing is that the sign of the function $\varphi(t)$ is allowed to be changed for $t \in[0, T]$.
\end{abstract}

Keywords: Rayleigh equation; topological degree; singularity; periodic solution

\section{Introduction}

The problem of a periodic solution for second ordinary differential equations with singularities has attracted much attention of many researchers because there are a great many applications of it from physics and mechanics (see [1-5] and the references therein). For example, the following second ordinary differential equation with singularity:

$$
x^{\prime \prime}(t)+c x^{\prime}(t)-\frac{1}{x^{\lambda}(t)}=e(t)
$$

is used for describing the motion of particles subject to Newtonian type forces or to restoring forces caused by compressed gases. Lazer and Solimini in a pioneering paper [6] first used the method of topological degree to study equation (1.1) for the case of $c=0$ and $\lambda \geq 1$. A necessary and sufficient condition for the existence of a positive periodic solution is that $\bar{e}:=\frac{1}{T} \int_{0}^{T} e(s) d s<0$. After that, the interest in the study of the existence of periodic solutions for second-order differential equations with singularities increased. In the past years, there was much work on the study of problem of periodic solutions for some second ordinary differential equations with singularities of repulsive type [7-20]. The problem of the existence of positive periodic solutions was extensively studied in [12-15] for the equation of conservative type,

$$
x^{\prime \prime}(t)+a(t) x-\frac{b(t)}{x^{\lambda}}=h(t),
$$

(0) The Author(s) 2017. This article is distributed under the terms of the Creative Commons Attribution 4.0 International License (http://creativecommons.org/licenses/by/4.0/), which permits unrestricted use, distribution, and reproduction in any medium, provided you give appropriate credit to the original author(s) and the source, provide a link to the Creative Commons license, and indicate if changes were made. 
where $a, b, h \in L^{1}[0, T]$ and $\lambda>0$. The crucial condition in [12-15] is that the function $a(t)$ is required to be

$$
a(t) \geq 0 \quad \text { for all } t \in[0, T] .
$$

By using a continuation theorem of Mawhin, Zhang in [17] considered the problem of periodic solutions of the Liénard equation with a singularity of repulsive type suggested by the fundamental example

$$
x^{\prime \prime}+f(x) x^{\prime}+\varphi(t) x-\frac{1}{x^{\lambda}}=h(t)
$$

where $\varphi, h \in L^{1}[0, T], f \in C([0,+\infty), R)$ and $\lambda \geq 1$. Wang in [18] extended equation (1.2) to the case of a delay singular equation,

$$
x^{\prime \prime}+f(x) x^{\prime}+\varphi(t) x(t-\tau)-\frac{1}{x^{\lambda}(t-\tau)}=h(t) .
$$

In $[17,18]$, the function $\varphi$ is required to be

$$
\varphi(t) \geq 0 \quad \text { for all } t \in[0, T] .
$$

However, there were few papers considering the periodic solutions for singular Rayleigh equations. To the best of our knowledge, the existence of positive periodic solutions was considered in [21] for a $p$-Laplacian Rayleigh equation with singularity of the form

$$
\left(\left|x^{\prime}\right|^{p-2} x^{\prime}\right)^{\prime}+f\left(x^{\prime}\right)-g_{1}(x)+g_{2}(x)=h(t)
$$

and

$$
\left(\left|x^{\prime}\right|^{p-2} x^{\prime}\right)^{\prime}+f\left(x^{\prime}\right)+g_{1}(x)-g_{2}(x)=h(t),
$$

where $p>1$ is a constant, $f: \mathbb{R} \rightarrow \mathbb{R}$ is an arbitrary continuous function, $g_{1}, g_{2}:(0, \infty) \rightarrow \mathbb{R}$ are all continuous and $g_{1}(x)$ is unbounded as $x \rightarrow 0^{+}, h: \mathbb{R} \rightarrow \mathbb{R}$ is a $T$-periodic continuous function. Obviously, equation (1.5) and equation (1.6) are all singular at $x=0$. The firstorder derivative term $f(x) x^{\prime}$ in equation (1.2) and equation (1.3) satisfies $\int_{0}^{T} f(x(t)) x^{\prime}(t) d t=$ 0 , which is crucial for obtaining a priori bounds of all the possible $T$-periodic solutions for equation (1.2) and equation (1.3). But the first-order derivative term in equation (1.5) and equation (1.6) is $f\left(x^{\prime}\right)$, generally, $\int_{0}^{T} f\left(x^{\prime}(t)\right) d t=0$ does not hold. The method for estimating a priori bounds of all the possible $T$-periodic solutions in [21] is different from the corresponding ones in $[4,17,18]$.

Motivated by this, in this paper, we study the existence of positive $T$-periodic solutions for the equation with a singularity of the repulsive type,

$$
x^{\prime \prime}+f\left(x^{\prime}\right)+\varphi(t) x-\frac{1}{x^{r}}=h(t)
$$


where $f: \mathbb{R} \rightarrow \mathbb{R}$ is an arbitrary continuous function, $\varphi, h: \mathbb{R} \rightarrow \mathbb{R}$ are $T$-periodic functions with $h \in L^{1}([0, T], \mathbb{R})$ and $\varphi \in C([0, T], \mathbb{R})$. The interesting thing is that the sign of the function $\varphi$ is allowed to be changeable for $t \in[0, T]$, which is not only essentially different from the corresponding ones in [12-15] but also essentially different from the case of (1.4) in $[17,18]$.

\section{Preliminary lemmas}

Throughout this paper, let $C_{T}=\{x \in C(\mathbb{R}, \mathbb{R}): x(t+T)=x(t)$ for all $t \in \mathbb{R}\}$ with the norm defined by $\|x\|_{\infty}=\max _{t \in[0, T]}|x(t)|$. For any $T$-periodic solution $y(t)$ with $y \in L^{1}([0, T], \mathbb{R})$, $y_{+}(t)$ and $y_{-}(t)$ denote $\max \{y(t), 0\}$ and $-\min \{y(t), 0\}$, respectively, and $\bar{y}=\frac{1}{T} \int_{0}^{T} y(s) d s$. Clearly, $y(t)=y_{+}(t)-y_{-}(t)$ for all $t \in \mathbb{R}$, and $\bar{y}=\overline{y_{+}}-\overline{y_{-}}$.

The following lemma is a consequence of Theorem 3.1 in [22].

Lemma 2.1 Assume that there exist positive constants $M_{0}, M_{1}$ and $M_{2}$ with $0<M_{0}<M_{1}$, such that the following conditions hold.

1. For each $\lambda \in(0,1]$, each possible positive T-periodic solution $x$ to the equation

$$
u^{\prime \prime}+\lambda f\left(u^{\prime}\right)+\lambda \varphi(t) u-\lambda \frac{1}{u^{r}}=\lambda h(t)
$$

satisfies the inequalities $M_{0}<x(t)<M_{1}$ and $\left|x^{\prime}(t)\right|<M_{2}$ for all $t \in[0, T]$.

2. Each possible solution $c$ to the equation

$$
\frac{1}{c^{r}}-f(0)-c \bar{\varphi}+\bar{h}=0
$$

satisfies the inequality $M_{0}<c<M_{1}$.

3. We have

$$
\left(\frac{1}{M_{0}^{r}}-f(0)-M_{0} \bar{\varphi}+\bar{h}\right)\left(\frac{1}{M_{1}^{r}}-f(0)-M_{1} \bar{\varphi}+\bar{h}\right)<0
$$

Then equation (1.7) has at least one T-periodic solution u such that $M_{0}<u(t)<M_{1}$ for all $t \in[0, T]$.

In order to study the existence of positive periodic solutions to equation (1.7), we list the following assumptions.

$\left(\mathrm{H}_{1}\right) \quad|f(x)| \leq a_{0}|x|^{\mu}+a_{1}, 0<\mu<1, a_{0}, a_{1}>0$.

$\left(\mathrm{H}_{2}\right)$ The function $\varphi(t)$ satisfies the following conditions:

$$
\int_{0}^{T} \varphi_{+}(s) d s>0, \quad \sigma:=\frac{\int_{0}^{T} \varphi_{-}(s) d s}{\int_{0}^{T} \varphi_{+}(s) d s} \in[0,1)
$$

and

$$
\sigma_{1}:=\frac{T^{\frac{1}{2}}}{1-\sigma}\left(\int_{0}^{T} \varphi_{+}(s) d s\right)^{\frac{1}{2}} \in(0,1) .
$$


Remark 2.1 If assumption $\left(\mathrm{H}_{2}\right)$ holds, then there are constants $D_{1}$ and $D_{2}$ with $0<D_{1}<D_{2}$ such that

$$
\frac{1}{x^{r}}-f\left(x^{\prime}\right)-\bar{\varphi} x+\bar{h}>0 \quad \text { for all } x \in\left(0, D_{1}\right)
$$

and

$$
\frac{1}{x^{r}}-f\left(x^{\prime}\right)-\bar{\varphi} x+\bar{h}<0 \quad \text { for all } x \in\left(D_{2}, \infty\right) .
$$

Now, we suppose that assumptions $\left(\mathrm{H}_{1}\right)$ and $\left(\mathrm{H}_{2}\right)$ hold, and we embed equation (1.7) into the following equation family with a parameter $\lambda \in(0,1]$ :

$$
x^{\prime \prime}+\lambda f\left(x^{\prime}\right)+\lambda \varphi(t) x-\lambda \frac{1}{x^{r}}=\lambda h(t) .
$$

Let

$$
\Omega=\left\{x \in C_{T}: x^{\prime \prime}+\lambda f\left(x^{\prime}\right)+\lambda \varphi(t) x-\lambda \frac{1}{x^{r}}=\lambda h(t), \lambda \in(0,1] ; x(t)>0, \forall t \in[0, T]\right\},
$$

and $M_{0}, A_{0}$ are all independent of $(\lambda, x) \in(0,1] \times \Omega$, and there is a positive integer $k_{0}$ such that

$$
k_{0} M \geq M_{0}
$$

where $M$ is a positive constant.

Lemma 2.2 Assume that assumptions $\left(\mathrm{H}_{1}\right)-\left(\mathrm{H}_{2}\right)$ hold, then there is an integer $k^{*}>k_{0}$ such that, for each function $u \in \Omega$, there is a point $t_{0} \in[0, T]$ satisfying

$$
u\left(t_{0}\right) \leq k^{*} M
$$

Proof If the conclusion does not hold, then for each $k>k_{0}$ there is a function $u_{k} \in \Omega$ satisfying

$$
u_{k}(t)>k M \quad \text { for all } t \in[0, T] .
$$

From the definition of $\Omega$, we see

$$
u_{k}^{\prime \prime}+\lambda f\left(u_{k}^{\prime}\right)+\lambda \varphi(t) u_{k}-\lambda \frac{1}{u_{k}^{r}}=\lambda h(t) .
$$

By integrating (2.3) over the interval $[0, T]$, we have

$$
\int_{0}^{T} f\left(u_{k}^{\prime}(t)\right) d t+\int_{0}^{T} \varphi_{+}(t) u_{k}(t) d t-\int_{0}^{T} \varphi_{-}(t) u_{k}(t) d t-\int_{0}^{T} \frac{1}{u_{k}^{r}(t)} d t=\int_{0}^{T} h(t) d t,
$$

i.e.,

$$
\int_{0}^{T} \varphi_{+}(t) u_{k}(t) d t=T \bar{h}+\int_{0}^{T} \varphi_{-}(t) u_{k}(t) d t+\int_{0}^{T} \frac{1}{u_{k}^{r}(t)} d t-\int_{0}^{T} f\left(u_{k}^{\prime}(t)\right) d t .
$$


Since $\varphi_{+}(t) \geq 0$ and $\varphi_{-}(t) \geq 0$ for all $t \in[0, T]$, it follows from the integral mean value theorem that there are two points $\xi, \eta \in[0, T]$ such that

$$
\begin{aligned}
u_{k}(\xi) \overline{\varphi_{+}} T & =u_{k}(\eta) \overline{\varphi_{-}} T+\int_{0}^{T} \frac{1}{u_{k}^{r}(t)} d t-\int_{0}^{T} f\left(u_{k}^{\prime}(t)\right) d t+T \bar{h} \\
& \leq T \overline{\varphi_{-}}\left\|u_{k}\right\|_{\infty}+\int_{0}^{T} \frac{1}{u_{k}^{r}(t)} d t+\int_{0}^{T}\left|f\left(u_{k}^{\prime}(t)\right)\right| d t+T|\bar{h}| .
\end{aligned}
$$

By assumption $\left(\mathrm{H}_{1}\right)$, we have

$$
u_{k}(\xi) \overline{\varphi_{+}} T \leq T|\bar{h}|+\overline{\varphi_{-}}\left\|u_{k}\right\|_{\infty} T+\frac{T}{k^{r} M^{r}}+\int_{0}^{T} a_{0}\left|u_{k}^{\prime}(t)\right|^{\mu} d t+\int_{0}^{T} a_{1} d t .
$$

Then

$$
\begin{aligned}
u_{k}(\xi) \overline{\varphi_{+}} & \leq|\bar{h}|+\bar{\varphi}_{-}\left\|u_{k}\right\|_{\infty}+\frac{1}{k^{r} M^{r}}+\frac{a_{0}}{T} \int_{0}^{T}\left|u_{k}^{\prime}(t)\right|^{\mu} d t+a_{1} \\
& \leq|\bar{h}|+\overline{\varphi_{-}}\left\|u_{k}\right\|_{\infty}+\frac{1}{k^{r} M^{r}}+\frac{a_{0}}{T}\left(\int_{0}^{T}\left|u_{k}^{\prime}(t)\right| d t\right)^{\mu} T^{1-\mu}+a_{1} \\
& \leq \overline{\varphi_{-}}\left\|u_{k}\right\|_{\infty}+\frac{a_{0}}{T^{\mu}}\left(\int_{0}^{T}\left|u_{k}^{\prime}(t)\right| d t\right)^{\mu}+\left(|\bar{h}|+\frac{1}{k^{r} M^{r}}+a_{1}\right),
\end{aligned}
$$

i.e.,

$$
u_{k}(\xi) \leq \frac{\overline{\varphi_{-}}}{\overline{\varphi_{+}}}\left\|u_{k}\right\|_{\infty}+\frac{a_{0}}{T^{\mu} \overline{\varphi_{+}}}\left(\int_{0}^{T}\left|u_{k}^{\prime}(t)\right| d t\right)^{\mu}+\frac{|\bar{h}|+\frac{1}{k^{r} M^{r}}+a_{1}}{\overline{\varphi_{+}}} .
$$

In view of the inequality

$$
\left\|u_{k}\right\|_{\infty} \leq u_{k}(\xi)+T^{\frac{1}{2}}\left(\int_{0}^{T}\left|u_{k}^{\prime}(t)\right|^{2} d t\right)^{\frac{1}{2}}
$$

it follows from (2.4) and the condition of $\sigma \in[0,1)$, which is in assumption $\left(\mathrm{H}_{2}\right)$, that

$$
\begin{aligned}
\left\|u_{k}\right\|_{\infty} \leq & \frac{\overline{\varphi_{-}}}{\overline{\varphi_{+}}}\left\|u_{k}\right\|_{\infty}+\frac{a_{0}}{T^{\mu} \overline{\varphi_{+}}}\left(\int_{0}^{T}\left|u_{k}^{\prime}(t)\right| d t\right)^{\mu} \\
& +\frac{|\bar{h}|+\frac{1}{k^{r} M^{r}}+a_{1}}{\overline{\varphi_{+}}}+T^{\frac{1}{2}}\left(\int_{0}^{T}\left|u_{k}^{\prime}(t)\right|^{2} d t\right)^{\frac{1}{2}} .
\end{aligned}
$$

Then

$$
\begin{aligned}
\left\|u_{k}\right\|_{\infty} \leq & \frac{1}{1-\sigma} \frac{a_{0}}{T^{\mu} \overline{\varphi_{+}}}\left(\int_{0}^{T}\left|u_{k}^{\prime}(t)\right| d t\right)^{\mu}+\frac{|\bar{h}|+\frac{1}{k^{r} M^{r}}+a_{1}}{(1-\sigma) \overline{\varphi_{+}}}+\frac{T^{\frac{1}{2}}}{1-\sigma}\left(\int_{0}^{T}\left|u_{k}^{\prime}(t)\right|^{2} d t\right)^{\frac{1}{2}} \\
\leq & \frac{1}{1-\sigma} \frac{a_{0}}{T^{\mu} \overline{\varphi_{+}}}\left(\int_{0}^{T} 1^{2} d t\right)^{\frac{\mu}{2}}\left(\int_{0}^{T}\left|u_{k}^{\prime}(t)\right|^{2} d t\right)^{\frac{\mu}{2}} \\
& +\frac{T^{\frac{1}{2}}}{1-\sigma}\left(\int_{0}^{T}\left|u_{k}^{\prime}(t)\right|^{2} d t\right)^{\frac{1}{2}}+\frac{|\bar{h}|+\frac{1}{k^{r} M^{r}}+a_{1}}{(1-\sigma) \overline{\varphi_{+}}}
\end{aligned}
$$




$$
\begin{aligned}
= & \frac{a_{0}}{(1-\sigma) T^{\frac{\mu}{2}} \overline{\varphi_{+}}}\left(\int_{0}^{T}\left|u_{k}^{\prime}(t)\right|^{2} d t\right)^{\frac{\mu}{2}}+\frac{|\bar{h}|+\frac{1}{k^{r} M^{r}}+a_{1}}{(1-\sigma) \overline{\varphi_{+}}} \\
& +\frac{T^{\frac{1}{2}}}{1-\sigma}\left(\int_{0}^{T}\left|u_{k}^{\prime}(t)\right|^{2} d t\right)^{\frac{1}{2}} .
\end{aligned}
$$

On the other hand, by multiplying (2.3) with $u_{k}(t)$, and integrating it over the interval $[0, T]$, we obtain

$$
\begin{aligned}
\int_{0}^{T}\left|u_{k}^{\prime}(t)\right|^{2} d t= & \lambda \int_{0}^{T} f\left(u_{k}^{\prime}(t)\right) u_{k}(t) d t-\lambda \int_{0}^{T} \frac{u_{k}(t)}{u_{k}^{r}(t)} d t \\
& +\lambda \int_{0}^{T} \varphi(t) u_{k}^{2}(t) d t-\lambda \int_{0}^{T} h(t) u_{k}(t) d t
\end{aligned}
$$

which together with the fact of $\frac{1}{x^{r}}>0$ for all $x>0$ gives

$$
\begin{aligned}
\int_{0}^{T}\left|u_{k}^{\prime}(t)\right|^{2} d t \leq & \lambda \int_{0}^{T} f\left(u_{k}^{\prime}(t)\right) u_{k}(t) d t+\lambda \int_{0}^{T} \varphi(t) u_{k}^{2}(t) d t-\lambda \int_{0}^{T} h(t) u_{k}(t) d t \\
\leq & \left\|u_{k}\right\|_{\infty}\left(\int_{0}^{T} a_{0}\left|u_{k}^{\prime}(t)\right|^{\mu} d t+a_{1} T\right)+\left\|u_{k}\right\|_{\infty}^{2} \overline{\varphi_{+}} T+\left\|u_{k}\right\|_{\infty} \overline{h_{-}} T \\
\leq & \left\|u_{k}\right\|_{\infty}\left[a_{1} T+a_{0} T^{1-\mu}\left(\int_{0}^{T}\left|u_{k}^{\prime}(t)\right| d t\right)^{\mu}\right] \\
& +\left\|u_{k}\right\|_{\infty}^{2} \overline{\varphi_{+}} T+\left\|u_{k}\right\|_{\infty} \overline{h_{-}} T \\
\leq & \left\|u_{k}\right\|_{\infty}\left[a_{1} T+a_{0} T^{1-\mu} T^{\frac{\mu}{2}}\left(\int_{0}^{T}\left|u_{k}^{\prime}(t)\right|^{2} d t\right)^{\frac{\mu}{2}}\right] \\
& +\left\|u_{k}\right\|_{\infty}^{2} \overline{\varphi_{+}} T+\left\|u_{k}\right\|_{\infty} \overline{h_{-}} T \\
= & \left\|u_{k}\right\|_{\infty}^{2} T \overline{\varphi_{+}}+\left[a_{1} T+a_{0} T^{1-\frac{\mu}{2}}\left(\int_{0}^{T}\left|u_{k}^{\prime}(t)\right|^{2} d t\right)^{\frac{\mu}{2}}+T \overline{h_{-}}\right]\left\|u_{k}\right\|_{\infty}
\end{aligned}
$$

i.e.,

$$
\begin{aligned}
\left(\int_{0}^{T}\left|u_{k}^{\prime}(t)\right|^{2} d t\right)^{\frac{1}{2}} \leq & \sqrt{T \overline{\varphi_{+}}}\left\|u_{k}\right\|_{\infty} \\
& +\left[a_{1} T+a_{0} T^{1-\frac{\mu}{2}}\left(\int_{0}^{T}\left|u_{k}^{\prime}(t)\right|^{2} d t\right)^{\frac{\mu}{2}}+T \bar{h}_{-}\right]^{\frac{1}{2}}\left\|u_{k}\right\|_{\infty}^{\frac{1}{2}}
\end{aligned}
$$

Substituting (2.5) into the above formula,

$$
\begin{aligned}
& \left(\int_{0}^{T}\left|u_{k}^{\prime}(t)\right|^{2} d t\right)^{\frac{1}{2}} \\
& \leq\left[\frac{a_{0}}{(1-\sigma) T^{\frac{\mu}{2}} \overline{\varphi_{+}}}\left(\int_{0}^{T}\left|u_{k}^{\prime}(t)\right|^{2} d t\right)^{\frac{\mu}{2}}+\frac{|\bar{h}|+\frac{1}{k^{r} M^{r}}+a_{1}}{(1-\sigma) \overline{\varphi_{+}}}\right. \\
& \left.\quad+\frac{T^{\frac{1}{2}}}{1-\sigma}\left(\int_{0}^{T}\left|u_{k}^{\prime}(t)\right|^{2} d t\right)^{\frac{1}{2}}\right] \times \sqrt{T \overline{\varphi_{+}}}
\end{aligned}
$$


Chen and Lu Advances in Difference Equations （2017） 2017:106

Page 7 of 14

$$
\begin{aligned}
& +\left[a_{1} T+a_{0} T^{1-\frac{\mu}{2}}\left(\int_{0}^{T}\left|u_{k}^{\prime}(t)\right|^{2} d t\right)^{\frac{\mu}{2}}+T \bar{h}_{-}\right]^{\frac{1}{2}} \\
& \times\left[\frac{a_{0}}{(1-\sigma) T^{\frac{\mu}{2}} \overline{\varphi_{+}}}\left(\int_{0}^{T}\left|u_{k}^{\prime}(t)\right|^{2} d t\right)^{\frac{\mu}{2}}+\frac{|\bar{h}|+\frac{1}{k^{r} M^{r}}+a_{1}}{(1-\sigma) \overline{\varphi_{+}}}\right. \\
& \left.+\frac{T^{\frac{1}{2}}}{1-\sigma}\left(\int_{0}^{T}\left|u_{k}^{\prime}(t)\right|^{2} d t\right)^{\frac{1}{2}}\right]^{\frac{1}{2}} \\
& \leq \frac{a_{0} T^{\frac{1-\mu}{2}}}{(1-\sigma) \sqrt{\overline{\varphi_{+}}}}\left(\int_{0}^{T}\left|u_{k}^{\prime}(t)\right|^{2} d t\right)^{\frac{\mu}{2}} \\
& +\frac{\sqrt{T}\left[|\bar{h}|+\frac{1}{k^{r} M^{r}}+a_{1}\right]}{(1-\sigma) \sqrt{\bar{\varphi}_{+}}}+\frac{T \sqrt{\overline{\varphi_{+}}}}{1-\sigma}\left(\int_{0}^{T}\left|u_{k}^{\prime}(t)\right|^{2} d t\right)^{\frac{1}{2}} \\
& +\left[\sqrt{a_{1} T+T \bar{h}_{-}^{-}}+\sqrt{a_{0} T^{1-\frac{\mu}{2}}}\left(\int_{0}^{T}\left|u_{k}^{\prime}(t)\right|^{2} d t\right)^{\frac{\mu}{4}}\right] \\
& \times\left[\sqrt{\frac{a_{0}}{(1-\sigma) T^{\frac{\mu}{2}} \bar{\varphi}_{+}}}\left(\int_{0}^{T}\left|u_{k}^{\prime}(t)\right|^{2} d t\right)^{\frac{\mu}{4}}\right. \\
& \left.+\frac{T^{\frac{1}{4}}}{\sqrt{1-\sigma}}\left(\int_{0}^{T}\left|u_{k}^{\prime}(t)\right|^{2} d t\right)^{\frac{1}{4}}+\sqrt{\frac{|\bar{h}|+\frac{1}{k^{T} M^{r}}+a_{1}}{(1-\sigma) \bar{\varphi}_{+}}}\right] \\
& =\frac{T \sqrt{\bar{\varphi}_{+}}}{1-\sigma}\left(\int_{0}^{T}\left|u_{k}^{\prime}(t)\right|^{2} d t\right)^{\frac{1}{2}} \\
& +\sqrt{\frac{a_{0} T^{\frac{3-\mu}{2}}}{1-\sigma}}\left(\int_{0}^{T}\left|u_{k}^{\prime}(t)\right|^{2} d t\right)^{\frac{1+\mu}{4}} \\
& +\left[\frac{a_{0} T^{\frac{1-\mu}{2}}}{(1-\sigma) \sqrt{\overline{\varphi_{+}}}}+\sqrt{a_{0} T^{1-\frac{\mu}{2}}} \sqrt{\frac{a_{0}}{(1-\sigma) T^{\frac{\mu}{2}} \overline{\varphi_{+}}}}\right]\left(\int_{0}^{T}\left|u_{k}^{\prime}(t)\right|^{2} d t\right)^{\frac{\mu}{2}} \\
& +\left[\sqrt{a_{1} T+T \overline{h_{-}}} \sqrt{\frac{a_{0}}{(1-\sigma) T^{\frac{\mu}{2}} \overline{\varphi_{+}}}}\right. \\
& \left.+\sqrt{a_{0} T^{1-\frac{\mu}{2}}} \sqrt{\frac{|\bar{h}|+\frac{1}{k^{r} M^{r}}+a_{1}}{(1-\sigma) \bar{\varphi}_{+}}}\right]\left(\int_{0}^{T}\left|u_{k}^{\prime}(t)\right|^{2} d t\right)^{\frac{\mu}{4}} \\
& +\sqrt{a_{1} T+T \bar{h}_{-}} \frac{T^{\frac{1}{4}}}{\sqrt{1-\sigma}}\left(\int_{0}^{T}\left|u_{k}^{\prime}(t)\right|^{2} d t\right)^{\frac{1}{4}}
\end{aligned}
$$

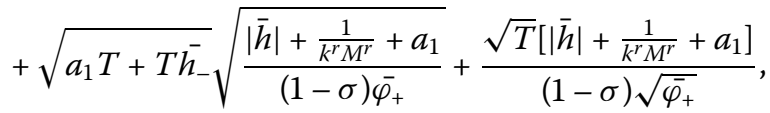

which results in

$$
\begin{aligned}
& {\left[1-\frac{T \sqrt{\bar{\varphi}_{+}}}{1-\sigma}\right]\left(\int_{0}^{T}\left|u_{k}^{\prime}(t)\right|^{2} d t\right)^{\frac{1}{2}}} \\
& \quad \leq \sqrt{\frac{a_{0} T^{\frac{3-\mu}{2}}}{1-\sigma}}\left(\int_{0}^{T}\left|u_{k}^{\prime}(t)\right|^{2} d t\right)^{\frac{1+\mu}{4}}+\sqrt{a_{1} T+T \bar{h}_{-}^{-}} \frac{T^{\frac{1}{4}}}{\sqrt{1-\sigma}}\left(\int_{0}^{T}\left|u_{k}^{\prime}(t)\right|^{2} d t\right)^{\frac{1}{4}}
\end{aligned}
$$




$$
\begin{aligned}
& +\left[\frac{a_{0} T^{\frac{1-\mu}{2}}}{(1-\sigma) \sqrt{\overline{\varphi_{+}}}}+\sqrt{a_{0} T^{1-\frac{\mu}{2}}} \sqrt{\frac{a_{0}}{(1-\sigma) T^{\frac{\mu}{2}} \overline{\varphi_{+}}}}\right]\left(\int_{0}^{T}\left|u_{k}^{\prime}(t)\right|^{2} d t\right)^{\frac{\mu}{2}} \\
& +\left[\sqrt{a_{1} T+T \overline{h_{-}}} \sqrt{\frac{a_{0}}{(1-\sigma) T^{\frac{\mu}{2}} \overline{\varphi_{+}}}}\right. \\
& \left.+\sqrt{a_{0} T^{1-\frac{\mu}{2}}} \sqrt{\frac{|\bar{h}|+\frac{1}{k^{r} M^{r}}+a_{1}}{(1-\sigma) \overline{\varphi_{+}}}}\right]\left(\int_{0}^{T}\left|u_{k}^{\prime}(t)\right|^{2} d t\right)^{\frac{\mu}{4}} \\
& +\sqrt{a_{1} T+T \overline{h_{-}}} \sqrt{\frac{|\bar{h}|+\frac{1}{k^{r} M^{r}}+a_{1}}{(1-\sigma) \overline{\varphi_{+}}}}+\frac{\sqrt{T}\left[|\bar{h}|+\frac{1}{(1-\sigma) \sqrt{k^{r} M^{r}}}+a_{1}\right]}{\left(1-\overline{\varphi_{+}}\right.} .
\end{aligned}
$$

It follows from assumption $\left(\mathrm{H}_{2}\right)$ that

$$
1-\frac{T \sqrt{\overline{\varphi_{+}}}}{1-\sigma}=1-\sigma_{1}>0
$$

which together with (2.7) shows that there is a constant $C_{0}$, which is independent of $\lambda$, such that

$$
\int_{0}^{T}\left|u_{k}^{\prime}(t)\right|^{2} d t \leq C_{0}
$$

Substituting (2.8) into (2.5), we have

$$
\left\|u_{k}\right\|_{\infty}<M_{0}
$$

Thus

$$
u_{k}(t)<M_{0} \quad \text { for all } t \in[0, T]
$$

By the definition of $k_{0}$, we see from (2.2) that (2.9) contradicts $u_{k}(t)>k M$ for all $t \in[0, T]$. This contradiction implies that the conclusion of Lemma 2.2 is true.

\section{Main results}

Theorem 3.1 Assume that assumptions $\left(\mathrm{H}_{1}\right)-\left(\mathrm{H}_{2}\right)$ hold, then equation (1.7) has at least one positive T-periodic solution.

Proof Firstly, we will show that there exist $M_{1}, M_{2}$ with $M_{1}>k^{*} M$ and $M_{2}>0$ such that each positive $T$-periodic solution $u(t)$ of equation (2.1) satisfies the inequalities

$$
u(t)<M_{1}, \quad\left|u^{\prime}(t)\right|<M_{2}, \quad \text { for all } t \in[0, T] .
$$

In fact, if $u$ is an arbitrary positive $T$-periodic solution of equation (2.1), then

$$
u^{\prime \prime}+\lambda f\left(u^{\prime}\right)+\lambda \varphi(t) u-\lambda \frac{1}{u^{r}}=\lambda h(t) .
$$

This implies $u \in \Omega$, so by using Lemma 2.2 we see that there is a point $t_{0} \in[0, T]$ such that

$$
u\left(t_{0}\right) \leq k^{*} M
$$


Chen and Lu Advances in Difference Equations （2017) 2017:106

Page 9 of 14

and then

$$
\|u\|_{\infty} \leq k^{*} M+T^{\frac{1}{2}}\left(\int_{0}^{T}\left|u^{\prime}(t)\right|^{2} d t\right)^{\frac{1}{2}} .
$$

Similar to the proof of (2.6), we have

$$
\begin{aligned}
\left(\int_{0}^{T}\left|u^{\prime}(t)\right|^{2} d t\right)^{\frac{1}{2}} \leq & \sqrt{T \overline{\varphi_{+}}}\|u\|_{\infty} \\
& +\left[a_{1} T+a_{0} T^{1-\frac{\mu}{2}}\left(\int_{0}^{T}\left|u^{\prime}(t)\right|^{2} d t\right)^{\frac{\mu}{2}}+T \bar{h}_{-}\right]^{\frac{1}{2}}\|u\|_{\infty}^{\frac{1}{2}} .
\end{aligned}
$$

Substituting (3.3) into (3.4), we have

$$
\begin{aligned}
\left(\int_{0}^{T}\left|u^{\prime}(t)\right|^{2} d t\right)^{\frac{1}{2}} \leq & \sqrt{T \overline{\varphi_{+}}}\left[k^{*} M+T^{\frac{1}{2}}\left(\int_{0}^{T}\left|u^{\prime}(t)\right|^{2} d t\right)^{\frac{1}{2}}\right] \\
& +\left[a_{1} T+a_{0} T^{1-\frac{\mu}{2}}\left(\int_{0}^{T}\left|u^{\prime}(t)\right|^{2} d t\right)^{\frac{\mu}{2}}+T \overline{h_{-}}\right]^{\frac{1}{2}} \\
& \times\left[k^{*} M+T^{\frac{1}{2}}\left(\int_{0}^{T}\left|u^{\prime}(t)\right|^{2} d t\right)^{\frac{1}{2}}\right]^{\frac{1}{2}} \\
\leq & T \sqrt{\overline{\varphi_{+}}}\left(\int_{0}^{T}\left|u^{\prime}(t)\right|^{2} d t\right)^{\frac{1}{2}}+\sqrt{T \overline{\varphi_{+}} k^{*} M} \\
& +\left[\sqrt{a_{0} T^{1-\frac{\mu}{2}}}\left(\int_{0}^{T}\left|u^{\prime}(t)\right|^{2} d t\right)^{\frac{\mu}{4}}+\sqrt{a_{1} T+T \overline{h_{-}}}\right] \\
& \times\left[\sqrt{k^{*} M}+T^{\frac{1}{4}}\left(\int_{0}^{T}\left|u^{\prime}(t)\right|^{2} d t\right)^{\frac{1}{4}}\right] \\
= & T \sqrt{\overline{\varphi_{+}}}\left(\int_{0}^{T}\left|u^{\prime}(t)\right|^{2} d t\right)^{\frac{1}{2}}+\sqrt{a_{0} T^{\frac{3-\mu}{2}}}\left(\int_{0}^{T}\left|u^{\prime}(t)\right|^{2} d t\right) \\
& +\sqrt{k^{*} M a_{0} T^{1-\frac{\mu}{2}}}\left(\int_{0}^{T}\left|u^{\prime}(t)\right|^{2} d t\right)^{\frac{\mu+\mu}{4}} \\
& +\sqrt{\left(a_{1}+\overline{h_{-}}\right) T^{\frac{3}{2}}}\left(\int_{0}^{T}\left|u^{\prime}(t)\right|^{2} d t\right)^{\frac{1}{4}} \\
& +\sqrt{k^{*} M\left(a_{1} T+T \overline{h_{-}}\right)+\sqrt{T \overline{\varphi_{+}} k^{*} M,}}
\end{aligned}
$$

which results in

$$
\begin{aligned}
{[1-} & T \sqrt{\left.\overline{\varphi_{+}}\right]}\left(\int_{0}^{T}\left|u^{\prime}(t)\right|^{2} d t\right)^{\frac{1}{2}} \\
\leq & \sqrt{a_{0} T^{\frac{3-\mu}{2}}}\left(\int_{0}^{T}\left|u^{\prime}(t)\right|^{2} d t\right)^{\frac{1+\mu}{4}}+\sqrt{a_{1} T+T \bar{h}_{-}^{-}} T^{\frac{1}{4}}\left(\int_{0}^{T}\left|u^{\prime}(t)\right|^{2} d t\right)^{\frac{1}{4}} \\
& +\sqrt{k^{*} M a_{0} T^{1-\frac{\mu}{2}}}\left(\int_{0}^{T}\left|u^{\prime}(t)\right|^{2} d t\right)^{\frac{\mu}{4}} \\
& +\sqrt{k^{*} M\left(a_{1} T+T \overline{h_{-}}\right)}+\sqrt{T \overline{\varphi_{+}}} k^{*} M .
\end{aligned}
$$


Since

$$
T \sqrt{\bar{\varphi}_{+}}=T^{\frac{1}{2}}\left(\int_{0}^{T} \varphi_{+}(t) d t\right)^{\frac{1}{2}}<\frac{T^{\frac{1}{2}}}{1-\sigma}\left(\int_{0}^{T} \varphi_{+}(t) d t\right)^{\frac{1}{2}}
$$

it follows from assumption $\left(\mathrm{H}_{2}\right)$ that

$$
1-T \sqrt{\bar{\varphi}_{+}}>0
$$

which together with (3.5) shows that there is a constant $\rho>0$, which is independent of $\lambda$, such that

$$
\left(\int_{0}^{T}\left|u^{\prime}(t)\right|^{2} d t\right)^{\frac{1}{2}}<\rho
$$

and then by (3.3), we have

$$
u(t) \leq k^{*} M+T^{\frac{1}{2}} \rho:=M_{1}, \quad \text { for all } t \in[0, T]
$$

Now, if $u$ attains its maximum over $[0, T]$ at $t_{2} \in[0, T]$, then $u^{\prime}\left(t_{2}\right)=0$ and we deduce from (3.2) that

$$
u^{\prime}(t)=\lambda \int_{t_{2}}^{t}\left[-f\left(u^{\prime}(t)\right)-\varphi(t) u(t)+\frac{1}{u^{r}(t)}+h(t)\right] d t
$$

for all $t \in\left[t_{2}, t_{2}+T\right]$. Then

$$
\begin{aligned}
\left|u^{\prime}(t)\right| \leq & \lambda \int_{t_{2}}^{t_{2}+T}\left(a_{0}\left|u^{\prime}(t)\right|^{\mu}+a_{1}\right) d t+\lambda \int_{t_{2}}^{t_{2}+T} \varphi(t) u(t) d t \\
& +\lambda \int_{t_{2}}^{t_{2}+T} \frac{1}{u^{r}(t)} d t+\lambda \int_{t_{2}}^{t_{2}+T}|h(t)| d t \\
\leq & \lambda a_{1} T+\lambda a_{0} \int_{0}^{T}\left|u^{\prime}(t)\right|^{\mu} d t+\lambda|\bar{\varphi}| T\|u\|_{\infty}+\lambda \int_{0}^{T} \frac{1}{u^{r}(t)} d t+\lambda T|\bar{h}| \\
\leq & \lambda a_{1} T+\lambda a_{0} T^{1-\mu}\left(\int_{0}^{T}\left|u^{\prime}(t)\right| d t\right)^{\mu} \\
& +\lambda|\bar{\varphi}| T\|u\|_{\infty}+\lambda \int_{0}^{T} \frac{1}{u^{r}(t)} d t+\lambda T|\bar{h}| \\
\leq & \lambda a_{1} T+\lambda a_{0} T^{1-\mu} T^{\frac{\mu}{2}}\left(\int_{0}^{T}\left|u^{\prime}(t)\right|^{2} d t\right)^{\frac{\mu}{2}} \\
& +\lambda|\bar{\varphi}| T\|u\|_{\infty}+\lambda \int_{0}^{T} \frac{1}{u^{r}(t)} d t+\lambda T|\bar{h}| \\
\leq & \lambda a_{1} T+\lambda a_{0} T^{1-\frac{\mu}{2}} \rho^{\mu}+\lambda|\bar{\varphi}| T M_{1}+\lambda \int_{0}^{T} \frac{1}{u^{r}(t)} d t+\lambda T|\bar{h}| .
\end{aligned}
$$

Integrating (3.2) over the interval $[0, T]$, we have

$$
-\int_{0}^{T} \frac{1}{u^{r}(t)} d t+\int_{0}^{T} f\left(u^{\prime}(t)\right) d t+\int_{0}^{T} \varphi(t) u(t) d t=\int_{0}^{T} h(t) d t
$$


then

$$
\begin{aligned}
\int_{0}^{T} \frac{1}{u^{r}(t)} d t & =\int_{0}^{T} f\left(u^{\prime}(t)\right) d t+\int_{0}^{T} \varphi(t) u(t) d t-\int_{0}^{T} h(t) d t \\
& \leq a_{1} T+a_{0} T^{1-\mu}\left(\int_{0}^{T}\left|u^{\prime}(t)\right| d t\right)^{\mu}+|\bar{\varphi}| T\|u\|_{\infty}+T|\bar{h}| \\
& \leq a_{1} T+a_{0} T^{1-\frac{\mu}{2}}\left(\int_{0}^{T}\left|u^{\prime}(t)\right|^{2} d t\right)^{\frac{\mu}{2}}+|\bar{\varphi}| T\|u\|_{\infty}+T|\bar{h}| \\
& \leq a_{1} T+a_{0} T^{1-\frac{\mu}{2}} \rho^{\mu}+|\bar{\varphi}| T M_{1}+T|\bar{h}| .
\end{aligned}
$$

It follows from (3.7) that

$$
\left|u^{\prime}(t)\right| \leq 2 \lambda\left(a_{1} T+a_{0} T^{1-\frac{\mu}{2}} \rho^{\mu}+|\bar{\varphi}| T M_{1}+T|\bar{h}|\right)=\lambda M_{2}, \quad \text { for all } t \in[0, T],
$$

and then

$$
\left|u^{\prime}(t)\right|<M_{2}, \quad \text { for all } t \in[0, T] .
$$

From (3.8) and (3.10), we see that there is a point $t_{1} \in[0, T]$ such that

$$
u\left(t_{1}\right) \geq \gamma
$$

where $\gamma<k^{*} M$ is a positive constant, which is independent of $\lambda \in(0,1]$.

Below, we will show that there exists a constant $\gamma_{0} \in(0, \gamma)$, such that each positive $T$ periodic solution of equation (2.1) satisfies

$$
u(t)>\gamma_{0} \quad \text { for all } t \in[0, T] .
$$

Suppose that $u(t)$ is an arbitrary positive $T$-periodic solution of equation (2.1), and $t_{1}$ be determined in (3.11). Multiplying (3.2) by $u^{\prime}(t)$ and integrating it over the interval $\left[t_{1}, t\right]$ (or $\left.\left[t, t_{1}\right]\right)$, we get

$$
\frac{\left|u^{\prime}(t)\right|^{2}}{2}-\frac{\left|u^{\prime}\left(t_{1}\right)\right|^{2}}{2}+\lambda \int_{t_{1}}^{t} f\left(u^{\prime}\right) u^{\prime} d t-\lambda \int_{t_{1}}^{t} \frac{u^{\prime}}{u^{r}} d t+\lambda \int_{t_{1}}^{t} \varphi(t) u u^{\prime} d t=\lambda \int_{t_{1}}^{t} h(t) u^{\prime} d t,
$$

which yields the estimate

$$
\begin{aligned}
\lambda\left|\int_{u\left(t_{1}\right)}^{u(t)} \frac{1}{u^{r}} d u\right| \leq & \frac{\left|u^{\prime}(t)\right|^{2}}{2}+\frac{\left|u^{\prime}\left(t_{1}\right)\right|^{2}}{2}+\lambda \int_{t_{1}}^{t}\left|f\left(u^{\prime}\right)\right|\left|u^{\prime}\right| d t \\
& +\lambda \int_{t_{1}}^{t}\left|\varphi(t) u u^{\prime}\right| d t+\lambda \int_{t_{1}}^{t}\left|h(t) u^{\prime}\right| d t .
\end{aligned}
$$

From (3.9) we get

$$
\lambda\left|\int_{u\left(t_{1}\right)}^{u(t)} \frac{1}{u^{r}} d u\right| \leq \lambda M_{2}^{2}+\lambda \max _{\left|u^{\prime}\right| \leq M_{2}}\left|f\left(u^{\prime}\right)\right| T M_{2}+\lambda M_{1} M_{2} T|\bar{\varphi}|+\lambda M_{2} T|\bar{h}|,
$$


which gives

$$
\left|\int_{u\left(t_{1}\right)}^{u(t)} \frac{1}{u^{r}} d u\right| \leq M_{3}, \quad \text { for all } t \in\left[t_{1}, t_{1}+T\right]
$$

By $\int_{0}^{1} \frac{1}{u^{r}} d u=\infty$, and $u\left(t_{1}\right) \geq \gamma$, there exists $\gamma_{0} \in(0, \gamma)$ such that $\int_{\gamma_{0}}^{\gamma} \frac{1}{u^{r}} d u>M_{3}$. Therefore, if there is a $t^{*} \in\left[t_{1}, t_{1}+T\right]$ such that $u\left(t^{*}\right) \leq \gamma_{0}$, then

$$
\int_{u\left(t^{*}\right)}^{u\left(t_{1}\right)} \frac{1}{u^{r}} d u \geq \int_{\gamma_{0}}^{\gamma} \frac{1}{u^{r}} d u>M_{3}
$$

which contradicts (3.13). This contradiction shows that $u(t)>\gamma_{0}$ for all $t \in[0, T]$.

Let $m_{0}=\min \left\{D_{1}, \gamma_{0}\right\}$ and $m_{1}=\max \left\{D_{2}, M_{1}\right\}$ be two constants, then from (3.6) and (3.10), we see that each possible positive $T$-periodic solution $u$ satisfies

$$
m_{0}<u(t)<m_{1}, \quad\left|u^{\prime}(t)\right|<M_{2} .
$$

This implies that condition 1 and condition 2 of Lemma 2.1 are satisfied. Also, we can deduce from Remark 2.1 that

$$
\frac{1}{c^{r}}-f(0)-\bar{\varphi} c+\bar{h}>0 \quad \text { for } c \in\left(0, m_{0}\right]
$$

and

$$
\frac{1}{c^{r}}-f(0)-\bar{\varphi} c+\bar{h}<0 \quad \text { for } c \in\left[m_{1}, \infty\right)
$$

which results in

$$
\left(\frac{1}{m_{0}^{r}}-f(0)-m_{0} \bar{\varphi}+\bar{h}\right)\left(\frac{1}{m_{1}^{r}}-f(0)-m_{1} \bar{\varphi}+\bar{h}\right)<0 .
$$

So condition 3 of Lemma 2.1 holds. By using Lemma 2.1, we see that equation (1.7) has at least one positive $T$-periodic solution. The proof is complete.

Example Considering the following equation:

$$
x^{\prime \prime}(t)+\left(x^{\prime}(t)\right)^{3}-\frac{1}{x^{2}(t)}+a(1+2 \sin t) x(t)=\cos t,
$$

where $a \in(0,+\infty)$ is a constant. Corresponding to equation (1.7), we have $f(x)=x^{3}, \varphi(t)=$ $a(1+2 \sin t)$ and $h(t)=\cos t$. By simple calculating, we can verify that assumptions $\left(\mathrm{H}_{1}\right)-$ $\left(\mathrm{H}_{2}\right)$ are satisfied. Furthermore,

$$
\int_{0}^{T} \varphi_{+}(s) d s=\left(\frac{4 \pi}{3}+2 \sqrt{3}\right) a, \quad \int_{0}^{T} \varphi_{-}(s) d s=\left(2 \sqrt{3}-\frac{2 \pi}{3}\right) a,
$$

and then

$$
\sigma:=\frac{\int_{0}^{T} \varphi_{-}(s) d s}{\int_{0}^{T} \varphi_{+}(s) d s}=\frac{2 \sqrt{3}-\frac{2 \pi}{3}}{\frac{4 \pi}{3}+2 \sqrt{3}} \in(0,1)
$$


and

$$
\sigma_{1}:=\frac{T^{\frac{1}{2}}}{1-\sigma}\left(\int_{0}^{T} \varphi_{+}(s) d s\right)^{\frac{1}{2}}=\frac{\sqrt{a}}{\sqrt{2 \pi}}\left(\frac{4 \pi}{3}+2 \sqrt{3}\right)^{\frac{3}{2}} .
$$

If

$$
a<\frac{2 \pi}{\left(\frac{4 \pi}{3}+2 \sqrt{3}\right)^{3}}
$$

then $\sigma_{1} \in(0,1)$, this implies that assumption $\left(\mathrm{H}_{1}\right)$ holds. Thus, by using Theorem 3.1 , we see that equation (3.14) has at least one positive $2 \pi$-periodic solution.

Remark 3.1 Since the sign of $1+2 \sin t$ in $\varphi(t)$ is changing for $t \in[0, T]$, whether the balance condition in $[17,18]$ is satisfied remains unclear. So the conclusion of the example cannot be obtained by using the main results in $[17,18]$.

Competing interests

The authors declare that they have no competing interests.

\section{Authors' contributions}

All authors have equally contributed to obtaining new results in this article and also read and approved the final manuscript.

\section{Acknowledgements}

This research was supported by the NNSF of China (No: 11271197). The authors are very grateful to the referee for her/his careful reading of the original manuscript and for her/his valuable suggestions for improving this article.

\section{Publisher's Note}

Springer Nature remains neutral with regard to jurisdictional claims in published maps and institutional affiliations.

Received: 16 September 2016 Accepted: 8 March 2017 Published online: 08 April 2017

\section{References}

1. Lei, J, Zhang, MR: Twist property of periodic motion of an atom near a charged wire. Lett. Math. Phys. 60(1), 9-17 (2002)

2. Adachi, S: Non-collision periodic solutions of prescribed energy problem for a class of singular Hamiltonian systems. Topol. Methods Nonlinear Anal. 25, 275-296 (2005)

3. Hakl, R, Torres, PJ: On periodic solutions of second-order differential equations with attractive-repulsive singularities. J. Differ. Equ. 248, 111-126 (2010)

4. Jebelean, P, Mawhin, J: Periodic solutions of singular nonlinear perturbations of the ordinary p-Laplacian. Adv. Nonlinear Stud. 2(3), 299-312 (2002)

5. Zhang, M: Nonuniform nonresonance at the first eigenvalue of the $p$-Laplacian. Nonlinear Anal. 29(1), 41-51 (1997)

6. Lazer, AC, Solimini, S: On periodic solutions of nonlinear differential equations with singularities. Proc. Am. Math. Soc. 99, 109-114 (1987)

7. Tanaka, K: A note on generalized solutions of singular Hamiltonian systems. Proc. Am. Math. Soc. 122, 275-284 (1994)

8. Fonda, A: Periodic solutions for a conservative system of differential equations with a singularity of repulsive type. Nonlinear Anal. 24, 667-676 (1995)

9. Fonda, A, Manásevich, R, Zanolin, F: Subharmonic solutions for some second-order differential equations with a singularities. SIAM J. Math. Anal. 24, 1294-1311 (1993)

10. Terracini, S: Remarks on periodic orbits of dynamical systems with repulsive singularities. J. Funct. Anal. 111, 213-238 (1993)

11. Solimini, S: On forced dynamical systems with a singularity of repulsive type. Nonlinear Anal. 14, 489-500 (1990)

12. Torres, PJ: Weak singularities may help periodic solutions to exist. J. Differ. Equ. 232, 277-284 (2007)

13. Chu, J, Torres, PJ, Zhang, M: Periodic solutions of second order non-autonomous singular dynamical systems. J. Differ. Equ. 239, 196-212 (2007)

14. Li, X, Zhang, Z: Periodic solutions for second-order differential equations with a singular nonlinearity. Nonlinear Anal. 69, 3866-3876 (2008)

15. Jiang, D, Chu, J, Zhang, M: Multiplicity of positive periodic solutions to superlinear repulsive singular equations. J. Differ. Equ. 211, 282-302 (2005)

16. Martins, R: Existence of periodic solutions for second-order differential equations with singularities and the strong force condition. J. Math. Anal. Appl. 317, 1-13 (2006) 
17. Zhang, M: Periodic solutions of Lienard equations with singular forces of repulsive type. J. Math. Anal. Appl. 203, 254-269 (1996)

18. Wang, Z: Periodic solutions of Lienard equations with a singularity and a deviating argument. Nonlinear Anal. 16, 227-234 (2014)

19. Chu, J, Torres, PJ, Wang, F: Twist periodic solutions for differential equations with a combined attractive-repulsive singularity. J. Math. Anal. Appl. 437, 1070-1083 (2016)

20. Hakl, R, Torres, PJ, Zamora, M: Periodic solutions of singular second order differential equations: upper and lower functions. Nonlinear Anal. 74, 7078-7093 (2011)

21. Lu, S, Zhong, T, Chen, L: Periodic solutions for $p$-Laplacian Rayleigh equations with singularities. Bound. Value Probl. 2016, 96 (2016)

22. Manásevich, R, Mawhin, J: Periodic solutions for nonlinear systems with $p$-Laplacian-like operators. J. Differ. Equ. 145 367-393 (1998)

Submit your manuscript to a SpringerOpen ${ }^{\circ}$ journal and benefit from:

- Convenient online submission

Rigorous peer review

- Immediate publication on acceptance

Open access: articles freely available online

- High visibility within the field

- Retaining the copyright to your article 